\title{
REPRODUCING A DETECTION TEST FOR MAGNETIC TAPES DEGRADATION: ACETONE EXTRACTION TEST
}

\author{
Dr. Federica Bressan \\ Dr. Prof. Roberta Bertani \\ ${ }^{1}$ Dept. of Art History, Musicology and Theatre Studies, Ghent University, Belgium \\ ${ }^{2}$ Dept. of Industrial Engineering - Chemical sector, University of Padua, Italy
}

\begin{abstract}
Magnetic tape is an important medium in the capturing of information and has had widespread use in audio, video, and computer applications over the past 60 years. As years go by, the preservation of the information stored on tape is becoming of increasing concern to society, particularly as the carriers suffer from physical decay and they threaten the survival of recordings holding a great cultural and commercial value. This article presents the methodology and the results of a chemical test aimed to determine the state of degradation of magnetic tapes. The test can reveal the state of degradation of the polymeric chains composing the tape layers, which are subject to an irreversible process of degradation over time. This study contributes to the long-term goal of building a structured knowledge base about diagnostic tools and recovery methods for magnetic tapes.
\end{abstract}

Keywords: recovery methods, detection test, acetone extraction, magnetic tapes degradation, audio preservation, sound archives, digitization, multimedia cultural heritage, digital humanities

\section{INTRODUCTION}

The history of recorded sound normally dates back to the 1860 s, when the ÉdouardLéon Scott de Martinville invented the phonautograph with which he recorded the French folk song "Au Clair de la Lune". From Thomas Edison's mechanical phonograph patented in 1878 , to the "liquid" music we listen to today, a wealth of historical audio documents has been produced. This capital is normally stored on audio carriers, from the most obsolete like wax discs or cylinders (popular between the mid1890s to the mid-1910s), to the most recent like optical discs (e.g. the well known Compact Discs) or non audio specific carriers like Solid-State Drives (SSDs).

Audio heritage comprises a large part of our cultural legacy [1], influencing many aspects of our daily life: news reportages, scholarly studies especially those on intangible cultural heritage, including the performance practice of very popular music genres like jazz, where the training of young generations of musicians has invariably depended on the study of the recordings of the greats of the past. Memorable music productions have been recorded on tape, including international pop/rock hits as well as erudite compositions of "tape music" (the name derives from the physical manipulation that composers performed on the tapes in order to achieve the desired effects). Today, re-mastered editions (Legacy, Ultimate, etc.) of the original tapes are regularly released on the market with notable commercial success, for example "The Beatles Stereo Box Set" and "The Beatles Mono Box Set" in 2009. 
A serious threat endangers audio heritage: the physical decay of audio carriers, combined with the progressive disappearance of the equipment necessary to read them, and with the necessary knowledge to interpret the audio formats and to operate the equipment correctly. The degradation of audio carriers is mainly due to their intrinsic chemical instability [2], aggravated by inadequate handling and storage conditions. During the 1990s, a theoretical basis for the preservation of audio and visual heritage started developing. Today, the migration of the information embodied in the audiovisual carriers to new carriers, in an all-digital format, is the widely accepted solution to the problem of degrading carriers. However, diagnostic tools and recovery methods for historical audio carriers remains an open research field. Not only they would have a direct benefit on the tapes, allowing for better treatment planning, but also on the digitisation projects management, allowing for reliable estimates of the costs and timing of the intervention workflows. The Image Permanence Institute (IPI) at Rochester Institute of Technology, Rochester, New York carried out a survey in 17 among museums, libraries and archives that witnessed some major manifestations of decay in their tape collections [3, p.15]. A significant percentage of institutions have had experience with tape decay: around $80 \%$ have observed various decay symptoms and $35 \%$ have been unable to replay the tapes due to decay.

This article focuses on a chemical test, namely the acetone extraction test, aimed to determine the state of degradation of magnetic tapes. It is an interesting test method for its potential use in low-budget restoration projects, thanks to the almost immediate results (30-minute incubation) and the possibility to instruct non-experts to perform the test and interpret the results.

\section{BACKGROUND}

The critical concern in magnetic tapes degradation is the change in the physical properties, not the loss of magnetic characteristics [4]: magnetic tapes have proved to be rather stable in this aspect, and if a significant loss of magnetic characteristics is present, it usually is due to careless exposure to magnetic charges.

To date, a standard specification against which tape life can be evaluated is not available, and neither are standards on the life expectancy of hardware and on the problems associated with hardware wearing out or becoming obsolete [4]. The lack of diagnostic tools forces tape inspection to rely on visual examinations and on the examiner's expertise [3], producing evaluations that are descriptive at best ("in good condition", "some concerns", "obvious decaying", etc.). The situation is made worse by a rather fragmented approach to audio preservation, where individual studios or projects tend to implement the guidelines [5] independently, and moreover without making the methods and results public for example by means of peer-reviewed publications.

This article is part of a research agenda finalised to improve this situation. The authors wish to contribute to the normalisation of audio preservation practices around the world and facilitate the capitalisation of the patrimony represented by audio heritage. Experimental studies as well as project reports and the replication of previous experiments are equally important elements in the envisioned knowledge base the authors wish to contribute to. 


\section{REPRODUCIBILITY AND OPEN SCIENCE}

The experiment presented in this article was inspired by a previous study conducted in 2006 by the Image Permanence Institute (IPI) at Rochester Institute of Technology, Rochester, New York [3]. The authors' plan was to reproduce the study and then proceed with further analyses on the effects of thermal treatment (a common recovery method targeting tapes showing undesired shed, stickiness and squeal), but the samples showed an unexpected behaviour, that was not described in [3]. The discrepancy in the test observed effects is a reminder of the importance of reproducibility in science. Results presented in scientific publications should be verifiable and the described method should provide enough detail to allow replication, "reinforcing the transparency and accountability of research processes" [6, p.129]. The point of replication is not that of checking the correctness of other scientists' work, although this should always be allowed to discourage data tampering in a highly competitive professional field such as that in which scientists operate today. The point is that data must be "useful rather than simply available" [6, p.133], and re-usability is a key feature of any published data set. Actually, quite in contrast with the intent of checking others' work, re-usability allows scientists not to repeat previous work.

The Open Science movement has recently gained momentum among publishers, funders, institutions and practicing scientists across all areas of research. Open Science is about developing "the infrastructures, algorithms, terminologies and standards required to disseminate, visualise, retrieve and re-use data" [7, p.5]. Most importantly, Open Science is about the people who make science: the social aspect "is capitalised upon and upgraded to become an instrument of scientific work" [8, p.29].

In line with the spirit of Open Science, in this article we present the results obtained during the replication of the test conducted in 2006 and we plan future analyses to gain insight in the unexpected results (see Sec. Conclusions). The research agenda mentioned in the previous paragraph also embraces this spirit, in its methods and goals, i.e. scientific literary work and shared knowledge bases.

Figure 1. Tape samples ready for incubation (left); precision weighing balance $(0.0001 \mathrm{~g})$ used to weigh the samples before and after incubation.
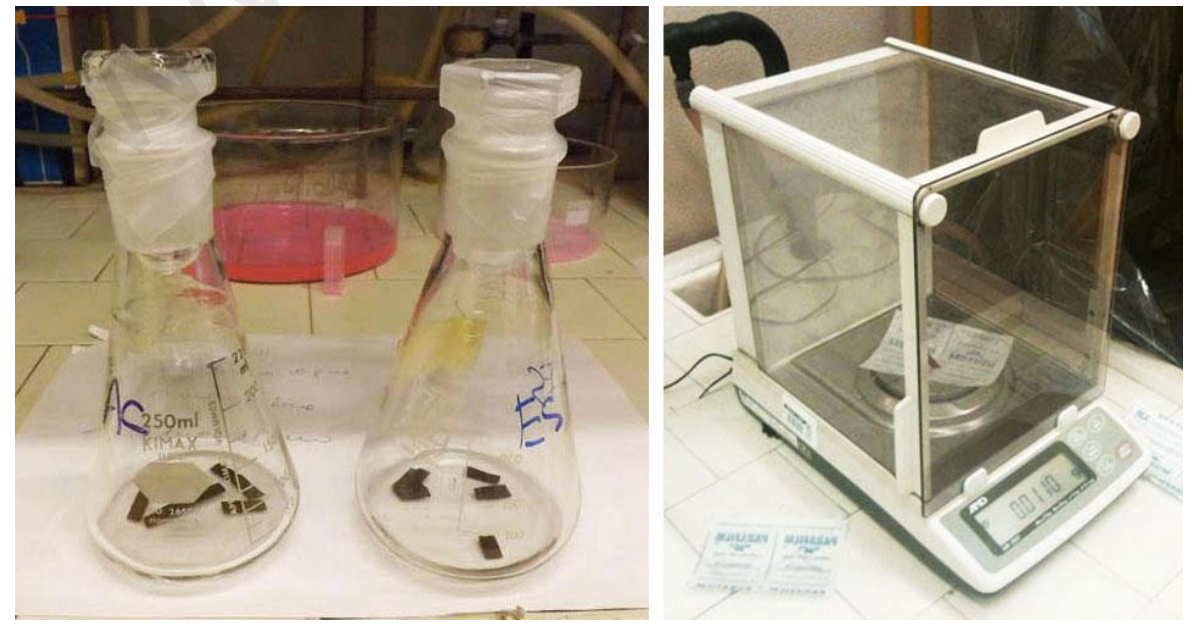


\section{ACETONE EXTRACTION TEST}

The test can reveal the state of degradation of the polymeric chains composing the tape layers, giving pointers for the adoption of precautionary measures at digitisation time. It provides information on tape binder stability (for a description of the layered structure of magnetic tapes, for example see [9]). The degradation products of the polyurethane binder are soluble in acetone, and the weight percent (wt.\%) of extractable is a measure of the degradation. Tape binder degradation is the result of polymer breakdown that occurs in reaction with humidity (i.e. hydrolysis). Hydrolytic breakdown causes a change in the structure of polymeric chains, producing low-molecular-weight fragments. These end-fragments are compounds that are mobile and tacky, and they are likely to be extractable in acetone. This test is designed to be performed during the first survey of an audio collection, in order to identify the percentage of damaged tapes and estimate the time and costs of the digitisation. The test can be summarised as follows: (1) a tape sample is weighed, (2) subsequently immersed in acetone for a given amount of time (in this study, 30 minutes); (3) finally, the acetone is adequately removed and the tape sample is weighed again. The eventual residue is ready for further analyses. Fig. 1 shows two sets of samples ready for incubation, and the precision weighing balance $(0.0001 \mathrm{~g})$ used to weigh the samples before and after incubation.

The acetone extraction test can be considered invasive and destructive in that a small tape sample needs to be cut from the reel. However, the sample size can be limited to 1 to 4 centimetres and can be taken from the tape head or tail, where normally no sound is recorded. Compared to the gain of having an indication of the health condition of the entire tape before even reading it on a reel-to-reel recorder, the loss appears justified except in the case of very rare or precious tapes. In fact, this evidences the importance of collecting a large number of case studies in a shared data base: a large pool of references would help make more accurate predictions on the tapes without testing them. The systematic accumulation of data and knowledge is a major goal in the authors' long-term commitment to the safeguard of audio heritage, in countertrend with a state of affairs where every estimate has to start from scratch or at best rely on the experts' experience - hence the wish to unlock the implicit knowledge currently embodied by the experts, give it a structure and make it available [10].

Table 1. Effect of acetone extraction time on the determination of percent acetone extractable. Average values were calculated based on four measurements for each test. The percent acetone extractable is expressed in wt.\% based on the weight loss of the tape sample. Source: [3, p. 26].

\begin{tabular}{|c|l|c|c|c|}
\hline \multicolumn{2}{|c|}{ Tape width } & \multicolumn{3}{|c|}{$\begin{array}{c}\text { \% acetone extractable obtained for vari- } \\
\text { ous extraction times }\end{array}$} \\
\cline { 3 - 5 } \multicolumn{2}{|c|}{} & $1 / 2$ hour & 1 hour & 2 hours \\
\hline \multirow{3}{*}{$3 / 4$ in } & Max. & 2.4 & 2.5 & 2.5 \\
\cline { 2 - 5 } & Min. & 2.1 & 2.3 & 2.3 \\
& Average & 2.2 & 2.4 & 2.4 \\
\hline \multirow{3}{*}{1 in } & Max. & 1.7 & 1.8 & 1.8 \\
\cline { 2 - 5 } & Min. & 1.6 & 1.7 & 1.8 \\
& Average & 1.6 & 1.7 & 1.8 \\
\hline
\end{tabular}




\section{Extraction time}

[3] reports that some preliminary tests show that the wt.\% of extractable is influenced by a variety of factors, most notably the duration of the acetone extraction. 30-minute immersion in acetone provides repeatable results. Shorter extraction times lead to inconsistent results, and longer extraction times do not significantly increase the amount of extractable compounds. Table 1 reports results obtained by [3] on 3/4 inch and 1-inch magnetic tape using 30-minute, one-hour, and two-hour extraction times in acetone. Average values were determined based upon four evaluations for each extraction time and tape width. It was shown that increasing the duration of acetone extraction beyond 30 minutes does not alter the outcome. The values determined for each set of four measurements conducted on each tape displayed negligible differences. Previous works [11] also suggest a 20-minutes extraction time. In the present study, the authors adopted a 30-minute extraction time. Based on the preliminary tests, [3] finalized an acetone extraction method, which was replicated herein. The method proved to provide reproducible results within an acceptable range. The data discussed in the following section were determined following the procedure summarised in Table 2.

Twelve tape samples have been analysed. The samples come from different historical audio collections: the exact year of production and the storage conditions are unknown, as is often the case with historical collections. All samples come from 1/4-inch wide tapes. Samples have been labelled sample A, sample B... to sample N.

\section{RESULTS AND DISCUSSION}

The values of the measurements for all tape samples are summarised in Table 3. Since the tape composition varies significantly between brands and models, and this determines their decay process and their reaction to the acetone immersion, the results of the characterisation analyses presented in [9] have also been included in Table 3.

The acetone extraction test was chosen based on its ability to measure an increasing proportion of extractable end-fragments from the polyurethane polymer as binder hydrolysis progresses. Measuring the percentage of extractable in acetone provides an indication of tape condition and reflects tape playability.

Table 3. Acetone extraction method used by [3] for testing magnetic tapes. Source: [3, p. 27].

\begin{tabular}{|l|l|}
\hline Step & Description \\
\hline 1. Sample preparation & $\begin{array}{l}\text { Sample weight: approx. } 0.5 \mathrm{mg} . \\
\text { Length of tape sample was based on tape width (e.g., 18" sample for 1" tape, 36" sample } \\
\text { for } 1 / 2 \text { " tape). } \\
\text { Four test samples were prepared for each tape tested. }\end{array}$ \\
\hline 2. Conditioning & Sample was conditioned to $21^{\circ} \mathrm{C}, 50 \% \mathrm{RH}$, for at least one hour. \\
\hline 3. Weighing & Sample was placed in a weighing bottle and weighed on precision scale $( \pm 0.0001 \mathrm{gram})$. \\
\hline 4. Acetone extraction & Sample was accordion-folded and immersed in $30 \mathrm{~mL}$ of acetone for 30 minutes. \\
\hline 5. Drying & $\begin{array}{l}\text { Sample was retrieved and rinsed in acetone. Then, it was placed on filter paper for } 15 \\
\text { minutes to drain and to let the acetone evaporate. Sample was placed in dry oven at } 50^{\circ} \mathrm{C} \\
\text { for } 15 \text { minutes. }\end{array}$ \\
\hline 6. Conditioning & Sample was conditioned to $21^{\circ} \mathrm{C}, 50 \%$ RH, for at least one hour. \\
\hline 7. Reweighing & Sample was placed in a weighing bottle and weighed on precision scale. \\
\hline 8. Calculation & $\begin{array}{l}\text { Acetone extractable was expressed in wt.\% based on weight loss of sample. } \\
\text { Final determination was expressed as average value based on four determinations for each } \\
\text { tape tested. }\end{array}$ \\
\hline
\end{tabular}


Figure 2. Tape samples A-L after the acetone extraction test. The wt.\% could only be measured for the samples F and H-N.

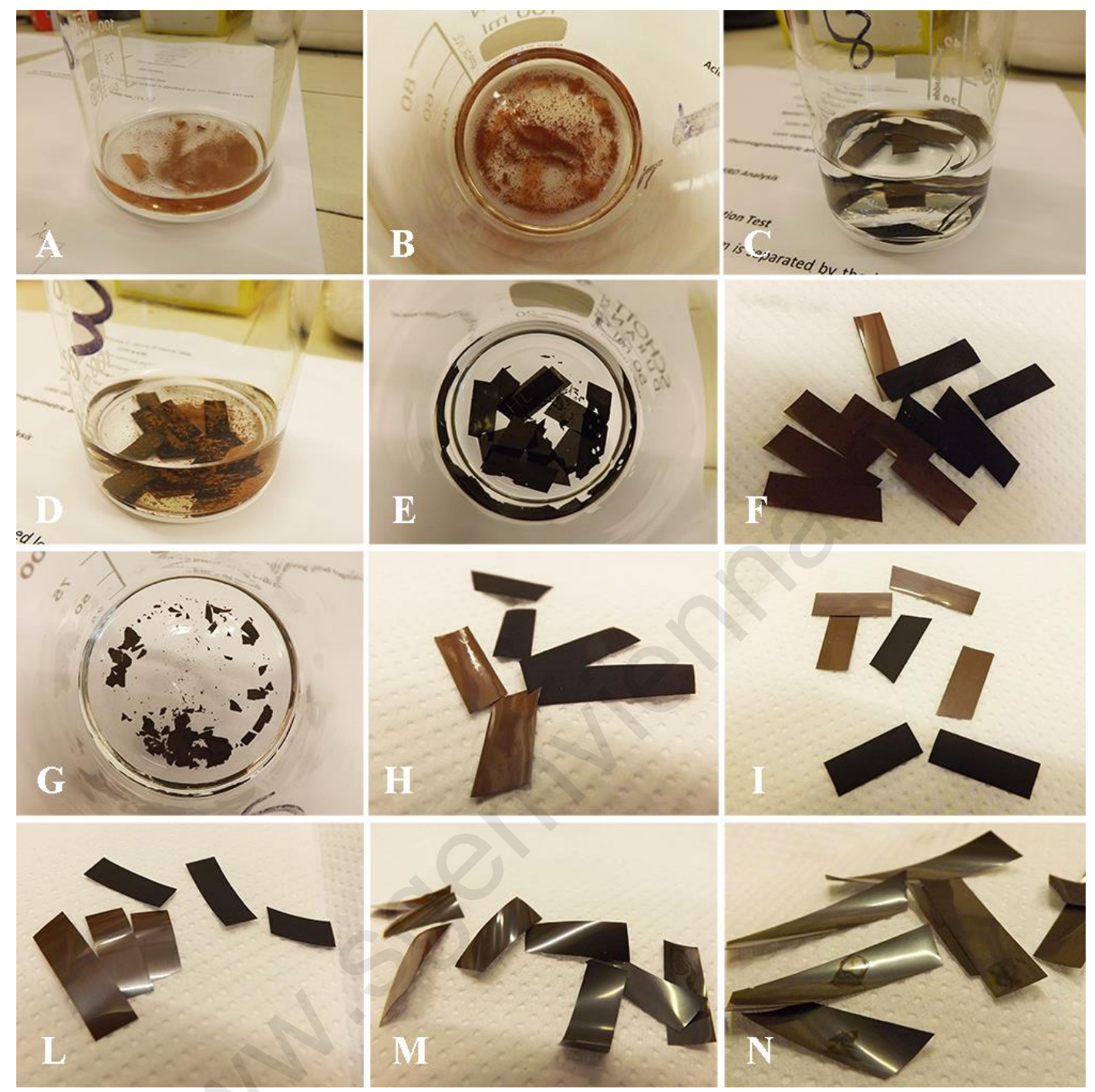

Such a measurement indirectly detects the presence of low-molecular-weight products and is a good indicator of either degraded or unstable polyurethane binder. Other tape components, such as lubricants, might also be soluble in acetone, however, and this may alter the results: for this reason, a study to analyse the composition of the residue has been planned next. Since tape formulation may vary significantly [12, p.60], it was expected that the wt.\% of extractable may also vary from one type of tape to another regardless of the degree of binder hydrolysis. Significant variation due to differences in format, manufacturer, or production batch was expected, besides a number of tape samples that were completely destroyed during the test, preventing any further measure of the weigh (Fig. 2). The number of tapes on which the test could not be performed due to this behaviour is significant $(50 \%)$. Table 3 specifies the type of outcome in the column on the far right: the weight loss is only specified for the samples that did not dissolve during the test, namely samples F and H-N. Fig. 2 shows how these samples 
remained fundamentally intact after the test, while the others disintegrated in different ways. This type of outcome was not reported in the study that inspired our experiment $[3, \mathrm{p} .25]$, nor in another work on the acetone extraction test documented in the scientific literature [13]. This does not only call for further investigation on the discrepant results and on the different ways in which the samples were destroyed during the test, but shows the importance of finding alternative tests for tapes on which the acetone extraction test cannot be performed (for example, acidity test, i.e. extraction in water).

Two of the tested samples were taken from tapes that were known to show symptoms of degradation like undesired shed, stickiness and squeal (samples I and L). The weight loss observed after the test is consistent with the literature about damaged tapes $(6.30 \%$ and $5.80 \%$, against an average $1.35 \%$ of the samples in better condition). This indicates that the magnetic coating is not perfectly adherent to the substrate.

\section{CONCLUSIONS AND FUTURE WORK}

This article reported the results of a detection test for the degradation of magnetic tapes, namely the acetone extraction test, inspired by a study conducted in 2006 [3]. The test is suitable to reveal the state of degradation of the polymeric chains constituting the tape layers, and is interesting for its potential application in low-budget preservation projects in (i) planning better treatments for magnetic tapes and (ii) more accurate estimates of the costs and timing of the digitisation workflow. However the results obtained in this study were found to be discrepant with those reported in the previous study. In the spirit Open Science, "encouraging critical thinking and ethical reflection among the researchers involved in data processing practices" [7, p.3], we plan to conduct more tests on a larger pool of samples, also in collaboration with the Istituto Superior Técnico in Lisbon, Portugal, with two goals: (i) to identify the reason why some samples disintegrate during the test, preventing the determination of their weight loss; (ii) to increase the size of the data base of case studies, in line with the research agenda aimed to structure, explicit and share the knowledge about diagnostic tools and recovery methods for magnetic tapes. A factor that slows down goal (ii) is that often times the tape composition of historical audio collections is not known and a range of other analyses is required to determine it [9].

Other tape components, such as lubricants, might also be soluble in acetone, however, and this may alter the results: for this reason, a study to analyse the composition of the residue has also been planned. The authors also plan to analyse the products of degradation by means of the acidity tests, in order to determine hydro-soluble substances, generally of acid nature, produced by hydrolysis. Another aspect to explore is the acidity of the tape surfaces by means of a chemical method developed at the Department of Industrial Engineering - Chemical sector, of the University of Padua.

\section{ACKNOWLEDGEMENTS}

This project has received funding from the European Union's Horizon 2020 research and innovation programme under the Marie Sklodowska-Curie grant agreement No. 703937. 
Table 3. Summary of the results of the acetone extraction test.

\begin{tabular}{|c|c|c|c|c|c|c|c|c|c|c|c|c|}
\hline 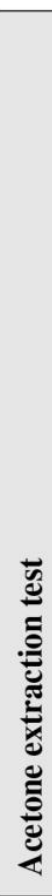 & 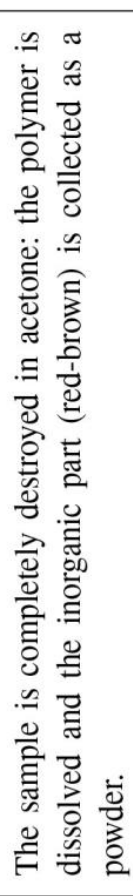 & 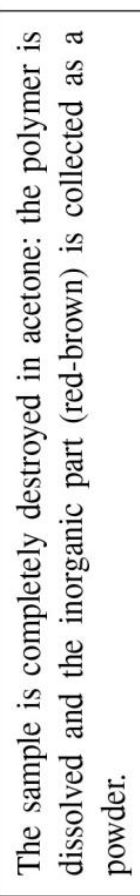 & 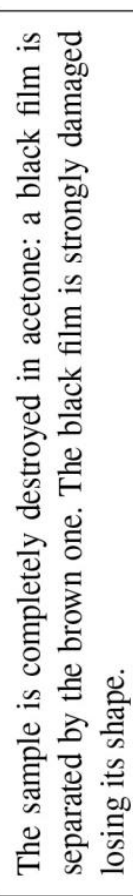 & 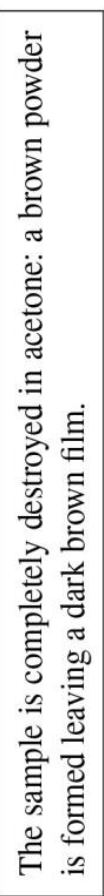 & 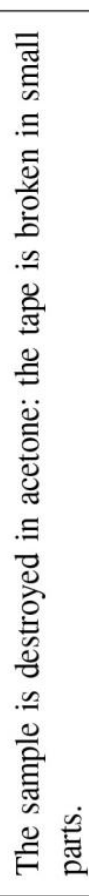 & 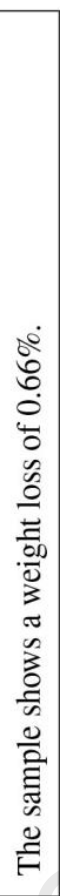 & 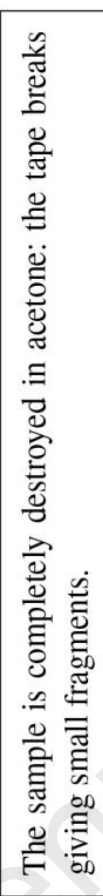 & 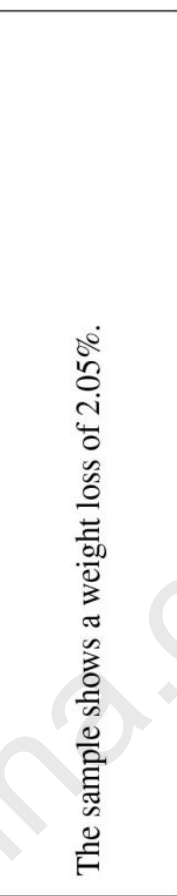 & 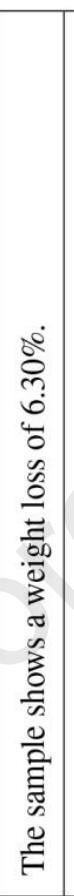 & 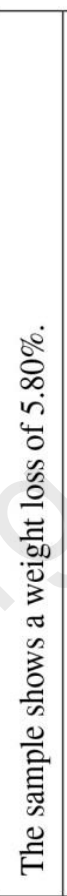 & 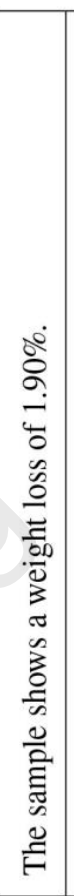 & 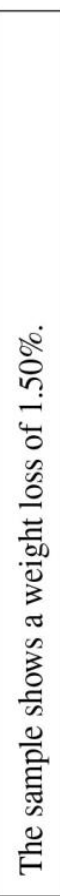 \\
\hline 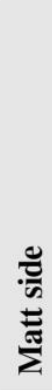 & 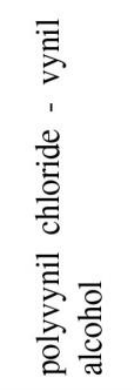 & 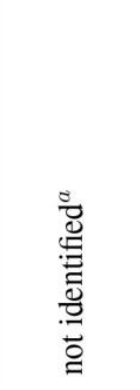 & 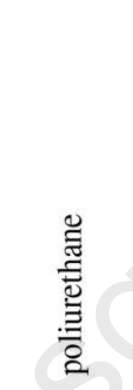 & $\begin{array}{l}\frac{\bar{u}}{\Delta} \\
\frac{2}{0} \\
\vdots\end{array}$ & 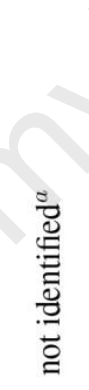 & 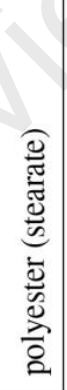 & 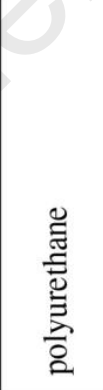 & 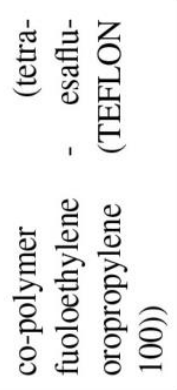 & 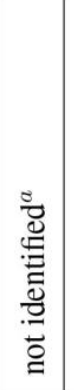 & 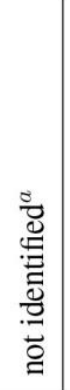 & 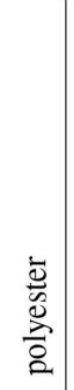 & 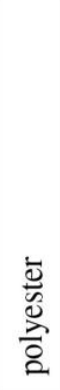 \\
\hline 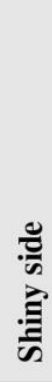 & 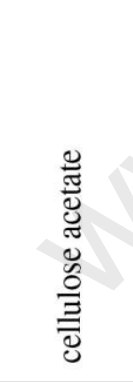 & 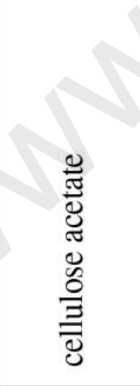 & 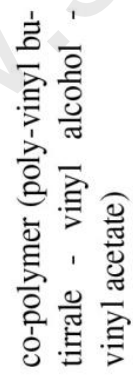 & 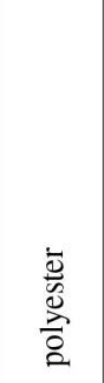 & 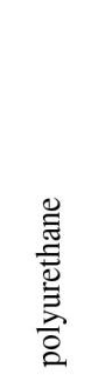 & 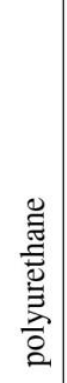 & $\frac{5}{2}$ & 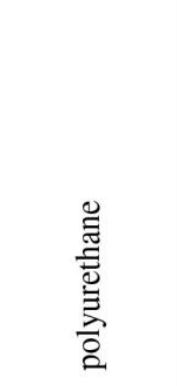 & 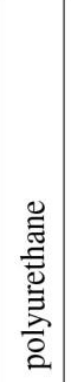 & 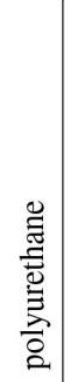 & 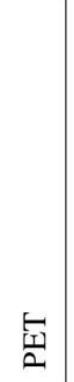 & $\sqrt[5]{2}$ \\
\hline 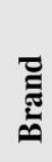 & 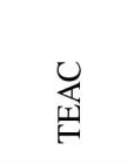 & 㺼 & $\begin{array}{l}\overrightarrow{1} \\
\frac{1}{x} \\
\Sigma\end{array}$ & 兰 & 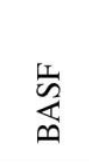 & 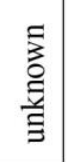 & $\sum_{m}$ & $\begin{array}{l}\frac{1}{5} \\
\frac{1}{m}\end{array}$ & 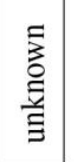 & $\begin{array}{l}\text { 寻 } \\
\text { 盖 }\end{array}$ & $\begin{array}{l}\overline{\bar{v}} \\
\text { x } \\
\sum^{\pi}\end{array}$ & $\begin{array}{l}\overline{\bar{v}} \\
\bar{x} \\
\sum_{\bar{z}}^{\tilde{z}}\end{array}$ \\
\hline 苂 & 忍 & 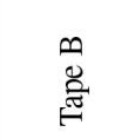 & $\begin{array}{l}0 \\
\text { Oे. } \\
\text { जu }\end{array}$ & 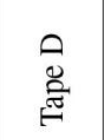 & 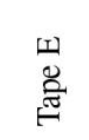 & 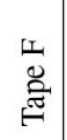 & 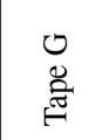 & 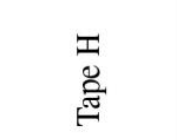 & 总 & 总 & 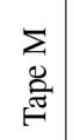 & $\begin{array}{l}z \\
0 \\
\text { 岕 } \\
\text { in }\end{array}$ \\
\hline
\end{tabular}




\section{REFERENCES}

[1] Edmonson R., Audiovisual Archiving: Philosophy and Principles. UNESCO, Bangkok, 2016.

[2] Schüller. D., The ethics of preserving audio and video documents. Technical report, UNESCO, Paris (France), 2006;

[3] Bigourdan J., Reilly J. M., Santoro K., and Salesin G., The preservation of magnetic tape collections: A perspective. Technical Report NEH GRANT PA-50123-03, Image Permanence Institute, Rochester Institute of Technology, Rochester, NY, 2006;

[4] Audio Engineering Society, AES recommended practice for audio preservation and restoration - Storage and handling - Storage of polyester-base magnetic tape. AES, New York, NY, 1997 (r2008).

[5] IASA Technical Committee, The Safeguarding of the Audiovisual Heritage: Ethics, Principles and Preservation Strategy, Co-Edited by Will Prentice and Lars Gaustad, Version 4, (Standards, Recommended Practices and Strategies, IASA-TC 03), International Association of Sound and Audiovisual Archives, 2017;

[6] Levin N., Leonelli S., Weckowska D., Castle D., and Dupré J., How do scientists define openness? Exploring the relationship between open science policies and research practice. Bulletin of Science, Technology and Society, 36(2), 2016, pp 128-14;

[7] Leonelli S., Locating ethics in data science: responsibility and accountability in global and distributed knowledge production systems. Philosophical Transactions of the Royal Society A, 374, 2016.

[8] Knorr-Cetina K., Epistemic Cultures: How the Sciences Make Knowledge. Harvard University Press, 1999.

[9] Bressan F., Bertani R., Furlan C., Simionato F., and Canazza S., An ATR FTIR and ESEM study on magnetic tapes for the assessment of the degradation of historical audio recordings. Journal of Cultural Heritage, 18, 2016, pp 313-320;

[10] Leonelli S. and Ankeny R. A., Repertoires: How to transform a project into a research community. Bio-Science, 65(7), 2015, pp 701-708;

[11] Bertram H. N. and Cuddihy E. F., Kinetics of the humid aging of magnetic recording tape. IEEE Transactions on Magnetics, 27, 1982, pp 4388-4395;

[12] Bressan F., Canazza S., and Bertani R., Honey, I burnt the tapes! A study on thermal treatment for the recovery of magnetic tapes affected by Sticky Shed Syndrome. IASA Journal, 44, 2015, pp 53-64;

[13] Cuddihy E., Aging of magnetic recording tape. IEEE Transactions on Magnetics, MAG-16(4), July 1980, pp 558-568; 